\title{
BMJ Open A phase 3 randomised study of enzalutamide plus leuprolide and enzalutamide monotherapy in high-risk non-metastatic hormone-sensitive prostate cancer with rising PSA after local therapy: EMBARK study design
}

\author{
Stephen J Freedland (D , ,,2 Ugo De Giorgi, ${ }^{1}$ Martin Gleave, ${ }^{4}$ Brad Rosbrook, \\ Qi Shen, ${ }^{6}$ Jennifer Sugg, ${ }^{7}$ Gabriel P Haas, ${ }^{8}$ Neal D Shore ${ }^{9}$
}

To cite: Freedland SJ, De Giorgi U, Gleave M, et al. A phase 3 randomised study of enzalutamide plus leuprolide and enzalutamide monotherapy in high-risk non-metastatic hormone-sensitive prostate cancer with rising PSA after local therapy: EMBARK study design. BMJ Open 2021;11:e046588. doi:10.1136/ bmjopen-2020-046588

- Prepublication history and additional supplemental material for this paper are available online. To view these files, please visit the journal online (http://dx.doi.org/10.1136/ bmjopen-2020-046588).

Received 17 November 2020 Accepted 14 June 2021

Check for updates

(c) Author(s) (or their employer(s)) 2021. Re-use permitted under CC BY-NC. No commercial re-use. See rights and permissions. Published by BMJ.

For numbered affiliations see end of article.

Correspondence to

Stephen J Freedland;

stephen.freedland@cshs.org

\section{ABSTRACT}

Introduction Limited data from controlled clinical trials are available for men who experience biochemical recurrence after definitive therapy for prostate cancer. In the absence of overt metastases, patients with nonmetastatic castration-sensitive prostate cancer (nmCSPC) often receive androgen deprivation therapy (ADT). There is no standard-of-care consensus on optimal ADT timing, although most men are treated prior to metastases, especially those with high-risk features (Gleason score 8-10 or prostate-specific antigen doubling time (PSADT) $<9-12$ months). Given data that ADT plus novel hormonal agents improve survival in men with metastatic CSPC, there is a desire to evaluate these agents earlier in the disease course. The main objective of EMBARK is the comparative assessment of enzalutamide plus leuprolide (luteinising hormone-releasing hormone agonist (LHRHa)) or enzalutamide monotherapy versus monotherapy LHRHa to improve metastasis-free survival (MFS) in patients with high-risk nmCSPC PSA recurrence after definitive therapy.

Methods and analysis EMBARK is a randomised, phase 3 study of high-risk patients with nmCSPC, a PSADT of $\leq 9$ months and a screening PSA of $\geq 2 \mathrm{ng} / \mathrm{mL}$ above the nadir after radiotherapy (RT) or $\geq 1 \mathrm{ng} / \mathrm{mL}$ after radical prostatectomy (RP) with or without postoperative RT. Men $(n=1050)$ are randomised 1:1:1 to enzalutamide $160 \mathrm{mg} /$ day plus LHRHa or placebo plus LHRHa (double-blind arms) or enzalutamide monotherapy (open-label arm). Treatment is suspended at week 37 if PSA concentrations are $<0.2 \mathrm{ng} / \mathrm{mL}$ and reinstated if levels rise to $\geq 2.0 \mathrm{ng} / \mathrm{mL}$ with RP or $\geq 5.0 \mathrm{ng} / \mathrm{mL}$ without RP. Patients with PSA $\geq 0.2 \mathrm{ng} / \mathrm{mL}$ at week 37 continue until treatment discontinuation criteria are met. The primary endpoint is MFS comparing enzalutamide plus LHRHa versus placebo plus LHRHa.

Ethics and dissemination The study is conducted under the guiding principles of the World Medical Association Declaration of Helsinki. The results will be disseminated at research conferences and in peer-reviewed journals. Trial registration number NCT02319837.
Strengths and limitations of this study

- EMBARK is the first study designed to determine whether early, combined therapy with enzalutamide plus a luteinising hormone-releasing hormone agonist (LHRHa) or enzalutamide monotherapy is more effective than placebo plus LHRHa in patients with high-risk non-metastatic castration-sensitive prostate cancer.

- A prostate-specific antigen (PSA) doubling time of $\leq 9$ months is included as a critical inclusion criterion based on its prior demonstration as a significant risk factor for prostate cancer-specific mortality and the primary endpoint of metastasisfree survival is a documented surrogate for overall survival in patients with localised disease.

- Monitoring PSA concentrations to inform treatment suspension in participants with undetectable PSA, and treatment continuation in those with detectable PSA, to evaluate whether intermittent androgen deprivation therapy (ADT) or an intermittent ADT holiday affords a clinical benefit together with modest improvements in quality of life, represents a principal feature of this protocol.

- A limitation of this study is the absence of biomarker analysis for study of enzalutamide response and resistance mechanisms.

- An additional study limitation is that some patients may develop non-metastatic castration-resistant prostate cancer before radiographic progression, based on prior PSA elevations, and discontinue their participation in the study.

\section{INTRODUCTION}

Background

Approximately one-third of patients experience biochemical recurrence (BCR; ie, prostate-specific antigen [PSA]-only recurrence) within 10 years after primary 
definitive therapy for prostate cancer. ${ }^{1-5}$ The rise in PSA concentration represents prostate cancer recurrence, as well as the likely presence of micrometastatic disease and an increased risk of prostate cancer-related morbidity and mortality. ${ }^{6}$ Patients with PSA doubling time (PSADT) $<9$ months are at high risk for rapid progression to radiologically evident metastases and eventual death. ${ }^{7-9}$

Treatments are limited for patients with high-risk nonmetastatic castration-sensitive prostate cancer (nmCSPC) with evidence of disease recurrence by PSA but without overt metastases. Standard of care options include systemic treatment with androgen deprivation therapy (ADT; orchiectomy or luteinising hormone-releasing hormone agonist [LHRHa] or LHRH antagonist), salvage local therapy, usually with radiotherapy (RT) or observation. ${ }^{6}$ For these patients, there is no general clinical consensus on optimal ADT timing either with early treatment to delay progression and hopefully prolong survival or with later treatment once metastases and symptoms develop to lessen the risk of adverse effects. ${ }^{10}$ Given limited data that early ADT may delay progression to metastases in high-risk patients exhibiting high-grade disease (eg, Gleason score of 8-10 or serum PSADT of $<12$ months), ${ }^{11}$ this approach is commonly employed for high-risk men. For patients who have exhausted local treatment options, a recent guideline from the American Urological Association (AUA), American Society for Radiation Oncology (ASTRO) and Society for Urologic Oncology recommends against routinely initiating ADT and using it as intermittent therapy if initiated. Guideline recommendations also call for observation or clinical trial enrolment. $^{12}$

Rather than continuous ADT, another option is continuous versus intermittent androgen blockade (IAD), although the latter is considered non-inferior to continuous ADT while offering modest quality-of-life (QoL) improvements in patients with nmCSPC. ${ }^{13}$ Finally, there is no general consensus for the use of ADT alone versus ADT plus a first-generation, non-steroidal antiandrogen (bicalutamide, flutamide and nilutamide), known as combined androgen blockade $(\mathrm{CAB})$, in patients with nmCSPC. American Society of Clinical Oncology (ASCO) guidelines suggest that $\mathrm{CAB}$ be considered in this setting, with personalised patient/physician treatment decisions in light of potential adverse effects and associated cost concerns. ${ }^{14}$

In an open-label, single-arm, phase 2 study of patients with nmCSPC and metastatic CSPC (mCSPC), treatment with enzalutamide monotherapy led to a rapid and durable response, with $92.5 \%$ of patients having a PSA decline of $\geq 80 \%$ at 25 weeks. ${ }^{15}$ PSA response was maintained with a favourable tumour response and was well tolerated at subsequent 1-year, ${ }^{16}$ 2-year ${ }^{16}$ and 3-year ${ }^{17}$ open-label follow-ups. While promising, no phase 3 study has yet tested enzalutamide monotherapy. Given data that $\mathrm{ADT}$ and novel hormonal agents improve survival and/or radiographic progression-free survival in men with $\mathrm{mCSPC}$, there is a desire to further evaluate such a combination even earlier in the disease course in a phase 3 study. ${ }^{18-20}$

\section{Rationale}

EMBARK is designed to provide further evidence to address whether treatment intensification by enzalutamide in the disease continuum (prior to the onset of metastasis or symptoms) is associated with improved metastasis-free survival (MFS) for men with high-risk nmCSPC and rising PSA concentrations after definitive therapy (figure 1). Treatment with enzalutamide has shown robust effects across the prostate cancer continuum, including in patients with mCSPC (ARCHES ${ }^{18}$ and ENZAMET ${ }^{21}$ ), patients with nmCRPC (PROSPER) ${ }^{22} 23$ and patients with metastatic castration-resistant prostate cancer (PREVAIL ${ }^{24-26}$ [chemotherapy naïve] and AFFIRM ${ }^{27}$ [postchemotherapy]), supporting the expectation of a significant treatment effect in men with nmCSPC. This phase 3 randomised study will determine whether administration of enzalutamide plus LHRHa or enzalutamide monotherapy is more effective than placebo plus LHRHa earlier along the prostate cancer continuum for patients with high-risk nmCSPC and rising PSA levels after local therapy. The PSA values have been blinded from study investigators to ensure that metastatic events rather than periodic, serum PSA determinations guide in the clinical decision to change therapy.

We included a monotherapy arm based on the Tombal et al phase 2 study demonstrating a rapid and durable PSA response described above. ${ }^{15-17}$ EMBARK is therefore designed to provide additional evidence relating to the efficacy and safety of monotherapy as a rationale for avoiding adverse events associated with LHRHa therapy, including diabetes, ischaemic heart disease and osteoporosis, ${ }^{28-30}$ but moreover to assess the QoL benefits of monotherapy.

\section{METHODS AND ANALYSIS \\ Study design}

EMBARK is an international, randomised phase 3 study of enzalutamide plus LHRHa, enzalutamide monotherapy and placebo plus LHRHa in men with high-risk nmCSPC and rising PSA concentrations after radical prostatectomy (RP), RT or both. The study was initiated on 17 December 2014 with target enrolment achieved on 18 June 2018. Study completion is estimated for 19 September 2026. High-risk patients with BCR after prior definitive therapy are characterised as having a PSADT $\leq 9$ months and a screening PSA of $\geq 1 \mathrm{ng} / \mathrm{mL}$ for patients who underwent prior RP (with or without RT) and $\geq 2 \mathrm{ng} / \mathrm{mL}$ above the nadir for patients who received primary RT only. These parameters were reached based on careful consideration of several factors, including the AUA definition of BCR (ie, detectable PSA level of $\geq 0.2 \mathrm{ng} / \mathrm{mL}$, with a second confirmatory level $>0.2 \mathrm{ng} / \mathrm{mL}$ after surgery ${ }^{31} 32$ along with the need for PSA to rise sufficiently to calculate 


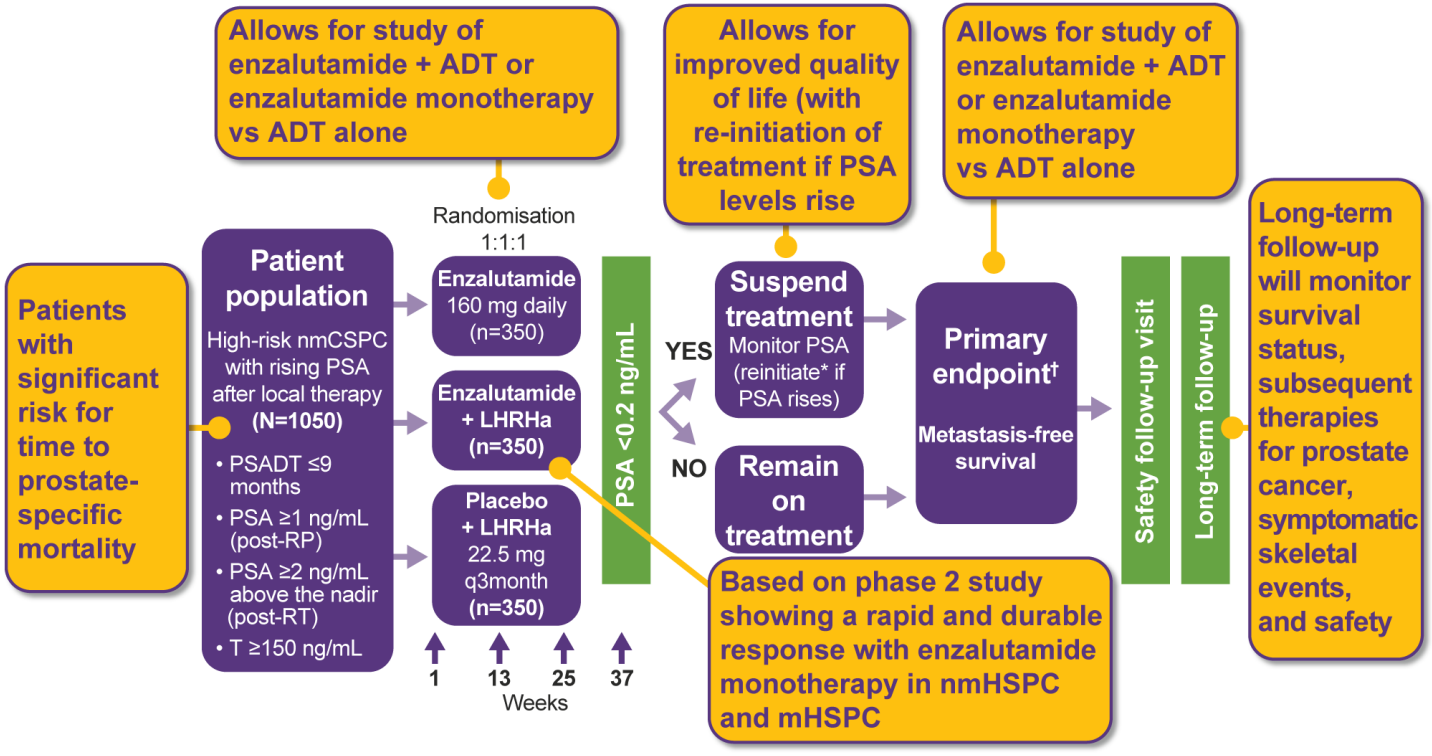

EMBARK was designed to address whether treatment intensification by use of novel hormonal therapy early in the prostate cancer disease continuum (prior to the onset of metastasis/symptoms) is associated with improved metastasis-free survival

Figure 1 EMBARK study design. *Study drug treatment reinitiated if PSA increases to $\geq 2.0 \mathrm{ng} / \mathrm{mL}$ for patients with prior prostatectomy or to $\geq 5.0 \mathrm{ng} / \mathrm{mL}$ for patients without prostatectomy. ${ }^{\dagger}$ For enzalutamide plus LHRHa versus placebo plus LHRHa, and secondary endpoint for enzalutamide monotherapy versus placebo plus LHRHa. ADT, androgen deprivation therapy; LHRHa, luteinising hormone-releasing hormone agonist; mHSPC, metastatic hormone-sensitive prostate cancer; nmCSPC, non-metastatic castration-sensitive prostate cancer; nmHSPC, non-metastatic hormone-sensitive prostate cancer; PSA, prostate-specific antigen; PSADT, PSA doubling time; T, testosterone.

an accurate PSADT. $^{33}$ Considering the association of elevated PSA levels with the onset of metastasis, a higher PSA cut-off would increase risk of metastases and need for ADT as standard of care prior to study eligibility. We therefore included patients with a short duration of ADT ( $\leq 6$ months prescribed for a rising PSA $\geq 9$ months prior to study entry). This decision also is based on findings of a median PSA level of $2.1 \mathrm{ng} / \mathrm{mL}$ at the time of ADT post-RP treatment failure in a multicentre Veteran's Administration cohort. ${ }^{34}$

Target enrolment was 1050 men with high-risk nmCSPC with rising PSA concentrations after RP, RT or both. No prior cytotoxic chemotherapy or ADT treatment $>6$ months for BCR was allowed. The primary efficacy endpoint is MFS.

\section{Patient and public involvement}

No patients were involved.

\section{Inclusion and exclusion criteria}

The inclusion criteria are as follows (box 1): (1) patients aged $\geq 18$ years; (2) histologically or cytologically confirmed adenocarcinoma of the prostate at initial biopsy, without neuroendocrine differentiation, signet cell or small cell features; (3) prostate cancer initially treated by RP, RT (including brachytherapy) or both, with curative intent; (4) PSADT $\leq 9$ months; (5) screening PSA by the central laboratory $\geq 1 \mathrm{ng} / \mathrm{mL}$ for participants who had RP (with or without RT) as primary treatment for prostate cancer and $\geq 2 \mathrm{ng} / \mathrm{mL}$ above the nadir for participants who had RT only as primary treatment for prostate cancer; (6) serum testosterone $\geq 150 \mathrm{ng} / \mathrm{dL}(5.2 \mathrm{nmol} / \mathrm{L})$ at screening and (7) Eastern Cooperative Oncology Group performance status of 0 or 1 at screening.

The exclusion criteria are as follows: (1) prior or present evidence of distant metastatic disease as seen on CT, MRI or bone scans; (2) prior hormonal therapy except for the following indications: neoadjuvant/adjuvant therapy to treat BCR $\leq 36$ months in duration and $\geq 9$ months before randomisation or a single dose or a short course ( $\leq 6$ months) of hormonal therapy given for rising PSA $\geq 9$ months before randomisation; (3) for patients who had prior RP, a suitable candidate for salvage RT as determined by the investigator per guidelines (eg, ASTRO/AUA, ${ }^{31}$ European Association of Urology $^{35}$ ); (4) prior cytotoxic chemotherapy, abiraterone acetate or enzalutamide for prostate cancer; (5) prior systemic biologic therapy, including immunotherapy, for prostate cancer; (6) history of seizure or any condition that may predispose to seizure and (7) clinically significant cardiovascular disease.

\section{Rationale for PSADT $\leq 9$ months as a critical inclusion criterion}

Previous data in a cohort of men who had undergone RP and developed subsequent BCR demonstrated that PSADT (as well as time to BCR and Gleason score) was a significant factor predictive of the probability and time to development of metastatic disease. ${ }^{7}$ To further stratify patients for risk of metastasis, a retrospective cohort study of patients 16 years after postprostatectomy BCR, reported that PSADT (<3.0 vs $3.0-8.9$ vs $9.0-14.9$ vs $\geq 15.0$ months), 


\section{Box 1 Eligibility criteria}

\section{Inclusion criteria}

$\rightarrow$ Aged $\geq 18$ years

- Histologically or cytologically confirmed adenocarcinoma of the prostate at initial biopsy, without neuroendocrine differentiation, signet cell or small cell features.

- Prostate cancer initially treated by radical prostatectomy (RP) or radiotherapy (RT; including brachytherapy) or both, with curative intent.

- Prostate-specific antigen (PSA) doubling time $\leq 9$ months.

- Screening PSA by the central laboratory $\geq 1 \mathrm{ng} / \mathrm{mL}$ for patients who had $\mathrm{RP}$ (with or without RT) as primary treatment for prostate cancer and $\geq 2 \mathrm{ng} / \mathrm{mL}$ above the nadir for patients who had only RT as primary treatment for prostate cancer.

- Serum testosterone $\geq 150 \mathrm{ng} / \mathrm{dL}(5.2 \mathrm{nmol} / \mathrm{L})$ at screening.

- Eastern Cooperative Oncology Group performance status of 0 or 1 at screening

\section{Exclusion criteria}

- Prior or present evidence of distant metastatic disease.

- Prior hormonal therapy. Neoadjuvant/adjuvant therapy to treat prostate cancer $\leq 36$ months in duration and $\geq 9$ months before randomisation or a single dose or a short course ( $\leq 6$ months) of hormonal therapy given for rising PSA $\geq 9$ months before randomisation is allowed.

- For patients who had a prior prostatectomy, a suitable candidate for salvage RT as determined by the investigator per guidelines (eg, American Society for Radiation Oncology/American Urological Association, ${ }^{31}$ European Association of Urology ${ }^{35}$ ).

- Prior cytotoxic chemotherapy, abiraterone acetate or enzalutamide for prostate cancer.

- Prior systemic biologic therapy, including immunotherapy, for prostate cancer.

- History of seizure or any condition that may predispose to seizure.

- Clinically significant cardiovascular disease.

Gleason score ( $\leq 7$ vs $8-10)$ and time from surgery to BCR ( $\leq 3$ vs $>3$ years) were all significant risk factors for time to prostate-specific mortality. ${ }^{8}$

\section{Dosage regimen}

Central randomisation (1:1:1) assigned study participants to one of the following treatment arms: enzalutamide plus LHRHa (double-blind); placebo plus LHRHa (doubleblind) or enzalutamide monotherapy (open-label). Enzalutamide is administered as $160 \mathrm{mg}$ /day by mouth with or without food. Leuprolide $22.5 \mathrm{mg}$ is administered as a single intramuscular or subcutaneous injection every 12 weeks.

\section{Rationale}

A key feature of the protocol is having a 1:1:1 randomisation that allows for the evaluation of monotherapy versus $\mathrm{ADT}$ as a secondary endpoint. This is of special interest as an open-label, single-arm, phase 2 study of patients with nmCSPC and mCSPC treated with enzalutamide monotherapy demonstrated that this treatment led to a rapid and durable PSA response. ${ }^{15-17}$ We are unaware of prior randomised, controlled trials comparing next-generation, oral antiandrogen monotherapy versus $\mathrm{ADT}$ in men with nmCSPC and PSA-only recurrence. Current ASCO guidelines support consideration of $\mathrm{CAB}$ in this setting but with individualised benefit-risk assessment in consideration of its increased costs and potential for greater adverse effects.

\section{Study procedures}

A central laboratory will quantify on-treatment PSA concentrations. With the exception of screening PSA values, PSA results will not be provided to study site investigators or participants. Alternatively, study sites will be notified if any PSA level meets a specified concentration threshold and a PSADT $\leq 10$ months while on study treatment. Imaging studies will be conducted every 6 months with CT or MRI to detect soft tissue disease and whole-body radionuclide bone scintigraphy (RBS) for bony metastasis. Serum PSA concentrations are monitored throughout the study (at screening, weeks 1, 25, 36, 37 and 49, repeating every 3 months until criteria are met for permanent treatment discontinuation [ie, signs of disease progression on conventional, radiographic imaging]), and study drug treatment is suspended at week 37 for participants whose PSA values are undetectable $(<0.2 \mathrm{ng} / \mathrm{mL})$ at week 36 . Study drug treatment may be suspended only once (at week 37 ) due to undetectable PSA and reinitiated if subsequent PSA levels increase to $\geq 2.0 \mathrm{ng} / \mathrm{mL}$ for participants with prior prostatectomy or $\geq 5.0 \mathrm{ng} / \mathrm{mL}$ for patients without prostatectomy. Participants with detectable PSA concentrations $(\geq 0.2 \mathrm{ng} / \mathrm{mL})$ at week 36 continue treatment without suspension until permanent treatment discontinuation criteria are met.

\section{Rationale}

A key feature of the protocol is monitoring PSA levels at week 36 and suspending study drug treatment at week 37 for participants with undetectable PSA $(<0.2 \mathrm{ng} / \mathrm{mL})$, while continuing study treatment for those with detectable PSA. The rationale for this aspect of the design is data, which demonstrate that IAD is non-inferior to continuous ADT for overall survival in nmCSPC. Intermittent androgen deprivation or an 'IAD treatment holiday' in patients with nmCSPC may afford clinical benefit together with modest improvements in QoL.

\section{Study endpoints}

The primary endpoint is MFS between enzalutamide plus LHRHa and placebo plus LHRHa (table 1).

\section{Rationale}

To benefit men with early-stage disease and features that indicate a high risk of morbidity and mortality from prostate cancer progression, a desirable therapy must demonstrate good efficacy in terms of delaying metastasis and death from prostate cancer, studied here using the defined primary endpoint of MFS, shown to be a surrogate of overall survival for patients with localised prostate cancer. ${ }^{36}$

A key secondary endpoint is MFS between enzalutamide monotherapy versus placebo plus LHRHa. 
Table 1 Objectives and endpoints

Primary objective

Primary endpoint

To evaluate the efficacy of enzalutamide plus LHRHa vs placebo MFS between enzalutamide plus LHRHa vs LHRHa plus LHRHa in patients with high-risk nmCSPC

\section{Key secondary objectives}

To evaluate efficacy of enzalutamide monotherapy vs placebo plus LHRHa

To compare enzalutamide plus LHRHa and enzalutamide alone vs placebo plus LHRHa in improving other efficacy measures

\section{Other secondary objectives}

To compare enzalutamide plus LHRHa and enzalutamide alone vs placebo plus LHRHa in improving other efficacy measures

\section{Secondary endpoints}

MFS between enzalutamide monotherapy vs placebo plus LHRHa

Time to PSA progression

Time to first use of antineoplastic therapy

Overall survival

\section{Other secondary endpoints}

Time to distant metastasis

Time to castration resistance

Time to symptomatic progression

Time to first symptomatic skeletal event (using the BPI-SF)

Time to clinically relevant pain

To compare enzalutamide plus LHRHa and enzalutamide alone vs placebo plus LHRHa based on PSA at week 36 (ie, whereby treatment is suspended at week 37 in participants with undetectable levels of $\leq 0.2 \mathrm{ng} / \mathrm{mL}$ )

\section{Proportion of participants per group who remain treatment-} free 2 years after suspension of study drug treatment at week 37 due to undetectable PSA

Proportion of participants per group with undetectable PSA 2 years after suspension of study drug treatment at week 37 due to undetectable PSA

Proportion of participants per group with undetectable PSA at 36 weeks on study drug

Time to resumption of any hormonal therapy following suspension at week 37 due to undetectable PSA

To compare PROs in enzalutamide plus LHRHa and enzalutamide alone arms vs placebo plus LHRHa arm PROs as measured by FACT-P, EQ-5D-5L and EORTC QLQPR25

To compare overall safety in enzalutamide plus LHRHa and enzalutamide alone arms vs placebo plus LHRHa arm

\section{Exploratory objective}

To compare progression-free survival after first subsequent therapy
Safety (adverse events, clinical laboratory tests, physical examinations and vital signs); monitored by independent data monitoring committee

\section{Exploratory endpoint}

Time from the date of randomisation to the first occurrence of investigator-determined disease progression

BPI-SF, Brief Pain Inventory-Short Form; EQ-5D-5L, EuroQol 5-Dimension 5-Level Health Assessment Instrument; FACT-P, Functional Assessment of Cancer Therapy-Prostate; LHRHa, luteinising hormone-releasing hormone agonist; MFS, metastasis-free survival; nmCSPC, non-metastatic castration-sensitive prostate cancer; PROs, patient-reported outcomes; PSA, prostate-specific antigen; EORTC QLQ-PR25, EORTC Quality of Life Questionnaire-Prostate 25.

\section{Rationale}

To assess the potential clinical benefit of enzalutamide monotherapy compared with LHRHa based on phase 2 data showing a rapid and durable PSA response with enzalutamide monotherapy. ${ }^{15-17}$

Other key secondary endpoints of enzalutamide plus LHRHa combination therapy or enzalutamide monotherapy versus placebo plus LHRHa are as follows: (1) time to PSA progression; (2) time to first use of antineoplastic therapy and (3) overall survival. Other secondary endpoints of enzalutamide plus LHRHa combination therapy or enzalutamide monotherapy versus placebo plus LHRHa are as follows: (1) time to distant metastasis; (2) proportion of participants per group who remain treatment-free 2 years after suspension of study drug at week 37 due to undetectable PSA; (3) proportion of participants per group with undetectable PSA 2 years after suspension of study drug at week 37 due to undetectable PSA; (4) proportion of participants per group with undetectable PSA at 36 weeks on study drug; (5) time to resumption of any hormonal therapy following study drug suspension at week 37 due to undetectable PSA; (6) time to castration resistance; (7) time to symptomatic progression; (8) time to first symptomatic skeletal event (SSE); (9) time to clinically relevant pain (assessed with the Brief Pain Inventory-Short Form [BPI-SF]); (10) QoL, based on Functional Assessment of Cancer TherapyProstate (FACT-P), EuroQol 5-Dimension 5-Level Health 
Assessment Instrument (EQ-5D-5L) and EORTC Quality of Life Questionnaire-Prostate 25 (EORTC QLQ-PR25) and (11) safety.

Exploratory endpoints include progression-free survival after first subsequent therapy, defined as time from the date of randomisation to the first occurrence of investigator-determined disease progression (PSA progression, progression on imaging or clinical progression) or death due to any cause, whichever occurred first, while the patient was receiving first subsequent therapy for prostate cancer.

\section{Efficacy and safety assessments}

Soft tissue disease is assessed by CT or MRI, with radiographic progression defined by Response Evaluation Criteria in Solid Tumours version 1.1. Bony metastasis is assessed by whole-body RBS, with radiographic progression defined as the appearance of one or more metastatic lesions on bone scan. Confirmation with a second imaging modality is required when lesions are detected in a single region on the bone scan. Appearance of metastatic lesions in two or more of the five regions on a bone scan does not require confirmation with a second imaging modality.

Other efficacy assessments include survival status, serum PSA values, serum testosterone concentrations, resumption of any hormonal therapy, new antineoplastic therapy, surgery/interventions for prostate cancer, SSEs and patient-reported outcomes (ie, BPI-SF, FACT-P, EQ-5D-5L, EORTC QLQ-PR25). The BPI-SF is a validated instrument using a self-reported scale to assess level of pain, its effects on activities of daily living and analgesic use. The short form contains nine, main, pain-related items rated on a severity and interference with activity scale of $0-10$, with 10 representing the worst pain. ${ }^{37}$

FACT-P is a self-reported, multidimensional QoL instrument specifically designed for use in men with prostate cancer. ${ }^{38}$ The questionnaire uses 27 core items to assess four domains of physical, social/family, emotional and functional well-being and 12 site-specific items to assess prostate-related symptoms. Each item is rated on a $0-4$ Likert-type scale and then combined to produce subscale scores for each domain as well as a global QoL score, with higher scores representing better QoL.

EQ-5D-5L is a standardised instrument that measures health-related QoL. ${ }^{39}$ Participants self-rate their current state of mobility, self-care, usual activities, pain/discomfort and anxiety/depression. They choose one of five possible responses that record level of severity (no problems, slight problems, moderate problems, severe problems or extreme problems) within each dimension. This tool also includes a visual analogue scale to describe general state of health from 'the worst health you can imagine' to 'the best health you can imagine'.

EORTC QLQ-PR25 is a module of the EORTC QLQ-30 questionnaire developed to assess the QoL of patients with prostate cancer. Participants self-rate their current state of pain as it relates to urination, ease and frequency of urination and bowel and other discomforts during the past week.
Participants also answer five questions on weight loss/gain and sexual interest and four questions about sexual activity during the past 4 weeks. Participants choose one of four possible responses that record level of intensity (not at all, a little, quite a bit, very much) within each dimension.

Safety assessments include adverse events, clinical laboratory tests, physical examinations, and vital signs.

Periodic monitoring of safety data as well as evaluation of interim efficacy results from this study will be conducted by an independent, external, Data Monitoring Committee of experts in prostate cancer, safety data monitoring, and statistics.

\section{Data analysis/statistical methods}

Statistical assumptions (MFS HR, 0.75) in the original EMBARK protocol were considered to be too conservative based on clinical trial results from SPARTAN ${ }^{40}$ and PROSPER. ${ }^{22}$ Therefore, the number of patients required for enrolment was reduced from 1860 to 1050 when the statistical plan was amended in June 2018. The study requires approximately 1050 participants to achieve the targeted total number of events, assuming a 30-month improvement in median MFS in the enzalutamide plus LHRHa group compared with the placebo plus LHRHa group. The primary efficacy analysis of MFS is conducted using the intention-to-treat population, defined as all participants randomly assigned to study treatment. Efficacy analyses incorporates the stratification factors applied at randomisation (screening PSA $\leq 10 \mathrm{ng} / \mathrm{mL}$ vs $>10 \mathrm{ng} / \mathrm{mL}$, PSADT $\leq 3$ months vs $>3$ to $\leq 9$ months and prior hormonal therapy vs no prior hormonal therapy). Treatment group comparisons are between the combination arms of enzalutamide plus LHRHa versus placebo plus LHRHa and between enzalutamide monotherapy versus placebo plus LHRHa. For the primary endpoint, MFS, the stratified log-rank test is employed to compare enzalutamide plus LHRHa versus placebo plus LHRHa. Treatment effect is estimated by HRs and 95\% CIs using a stratified Cox regression model. An interim analysis for efficacy/futility is planned.

\section{Ethics and dissemination}

The study is conducted under the guiding principles of the World Medical Association Declaration of Helsinki, including Good Clinical Practice according to International Council for Harmonisation Guidelines. Ethics committee approval will be obtained for extensive protocol amendments. All patients were required by study investigator to provide informed consent prior to start of the study (online supplemental file 1). Patient identify information will remain confidential as specified in the protocol or longer if required by local regulations. The results will be disseminated at several research conferences and as published articles in peer-reviewed journals after approval from the study sponsors.

\section{Author affiliations}

${ }^{1}$ Division of Urology, Department of Surgery, Samuel Oschin Comprehensive Cancer Institute, Cedars-Sinai Medical Center, Los Angeles, California, USA 
${ }^{2}$ Section of Urology, Durham VA Medical Center, Durham, North Carolina, USA ${ }^{3}$ Department of Medical Oncology, Istituto Scientifico Romagnolo per lo Studio e la Cura dei Tumori (IRST) IRCCS, Meldola, Italy

${ }^{4}$ Department of Urologic Sciences, Vancouver Prostate Centre, The University of British Columbia, Vancouver, British Columbia, Canada

${ }^{5}$ Department of Global Biometrics and Data Management, Pfizer, New York, New York, USA

${ }^{6}$ Department of Product Development, Pfizer, New York, New York, USA ${ }^{7}$ Department of Biostatistics, Astellas Pharma US, Northbrook, Illinois, USA ${ }^{8}$ Astellas Pharma Global Development, Northbrook, Illinois, USA

${ }^{9}$ Carolina Urologic Research Center, Myrtle Beach, South Carolina, USA

\section{Twitter Stephen J Freedland @SFreedlandMD}

Acknowledgements Dr Swetha Sridharan contributed to the protocol design. Contributors All authors have fulfilled authorship criteria. SJF, UDG, MG, BR, GPH and NDS contributed to protocol design and manuscript preparation. QS and JS contributed to protocol design and the statistical analysis plan.

Funding The EMBARK trial presented in this article is sponsored by Pfizer (New York, NY), and Astellas Pharma (Northbrook, IL), the codevelopers of enzalutamide (award/grant number is not applicable). Authors who are employed by the study sponsors, Pfizer and Astellas Pharma were involved in the design of the study, preparation of the manuscript and the decision to submit the manuscript for publication. Medical writing and editorial support funded by both sponsor companies were provided by Ira Mills, PhD and Dena McWain of Ashfield Healthcare Communications and Julie B Stimmel, PhD at Onyx (a Prime Global agency).

Competing interests SJF is a consultant to Astellas Pharma, Pfizer, Janssen, Bayer, Sanofi, Dendreon, Myovant, AstraZeneca and Merck. UDG is a consultant to Astellas Pharma, Bayer, BMS, Ipsen, Janssen, Novartis, Pfizer, Sanofi and Pharmamar; received institutional research funding from AstraZeneca, Roche and Sanofi and received travel funds from BMS, Ispen, Janssen, Pfizer and Roche during the conduct of the study. MG has stock or ownership interest in OncoGenex Technologies, Sustained Therapeutics and Sikta Pharmaceuticals; is a consultant to Astellas Pharma, AstraZeneca, Bayer, GDx, Janssen, Sanofi, Pfizer, Tersera and Roche; and holds patents for OGX-011, 0GX-427, ST-CP and ST-POP. BR is an employee of and holds stock ownership in Pfizer. QS is an employee of Pfizer. JS is an employee of Astellas Pharma with stock ownership in AstraZeneca. GPH is an employee of Astellas Pharma. NDS is a consultant to or received research funding from AbbVie, Amgen, Astellas Pharma, AstraZeneca, Bayer, BMS, Dendreon, Exact Sciences, Ferring, Fergene, Janssen, MDx Health, Merck, Myovant, Nymox, Pfizer, Sanofi and Tolmar.

Patient consent for publication Not required.

Provenance and peer review Not commissioned; externally peer reviewed.

Supplemental material This content has been supplied by the author(s). It has not been vetted by BMJ Publishing Group Limited (BMJ) and may not have been peer-reviewed. Any opinions or recommendations discussed are solely those of the author(s) and are not endorsed by BMJ. BMJ disclaims all liability and responsibility arising from any reliance placed on the content. Where the content includes any translated material, BMJ does not warrant the accuracy and reliability of the translations (including but not limited to local regulations, clinical guidelines, terminology, drug names and drug dosages), and is not responsible for any error and/or omissions arising from translation and adaptation or otherwise.

Open access This is an open access article distributed in accordance with the Creative Commons Attribution Non Commercial (CC BY-NC 4.0) license, which permits others to distribute, remix, adapt, build upon this work non-commercially, and license their derivative works on different terms, provided the original work is properly cited, appropriate credit is given, any changes made indicated, and the use is non-commercial. See: http://creativecommons.org/licenses/by-nc/4.0/.

\section{ORCID iD}

Stephen J Freedland http://orcid.org/0000-0002-8104-6419

\section{REFERENCES}

1 Amling CL, Blute ML, Bergstralh EJ, et al. Long-term hazard of progression after radical prostatectomy for clinically localized prostate cancer: continued risk of biochemical failure after 5 years. $J$ Urol 2000;164:101-5.

2 Bianco FJ, Scardino PT, Eastham JA. Radical prostatectomy: Longterm cancer control and recovery of sexual and urinary function ("trifecta"). Urology 2005;66:83-94.
3 Chun FK-H, Graefen M, Zacharias M, et al. Anatomic radical retropubic prostatectomy-long-term recurrence-free survival rates for localized prostate cancer. World J Urol 2006;24:273-80.

4 Han M, Partin AW, Pound CR, et al. Long-term biochemical disease-free and cancer-specific survival following anatomic radical retropubic prostatectomy. The 15-year Johns Hopkins experience. Urol Clin North Am 2001;28:555-65.

5 Liesenfeld L, Kron M, Gschwend JE, et al. Prognostic factors for biochemical recurrence more than 10 years after radical prostatectomy. J Urol 2017;197:143-8.

6 Mottet N, Bellmunt J, Bolla M, et al. EAU-ESTRO-SIOG guidelines on prostate cancer. Part 1: screening, diagnosis, and local treatment with curative intent. Eur Urol 2017;71:618-29.

7 Pound CR, Partin AW, Eisenberger MA, et al. Natural history of progression after PSA elevation following radical prostatectomy. JAMA 1999;281:1591-7.

8 Freedland SJ, Humphreys EB, Mangold LA, et al. Risk of prostate cancer-specific mortality following biochemical recurrence after radical prostatectomy. JAMA 2005;294:433-9.

9 Freedland SJ, Humphreys EB, Mangold LA, et al. Death in patients with recurrent prostate cancer after radical prostatectomy: prostatespecific antigen doubling time subgroups and their associated contributions to all-cause mortality. J Clin Oncol 2007;25:1765-71.

10 Garcia-Albeniz X, Chan JM, Paciorek A, et al. Immediate versus deferred initiation of androgen deprivation therapy in prostate cancer patients with PSA-only relapse. An observational follow-up study. Eur $J$ Cancer 2015;51:817-24.

11 Moul JW, Wu H, Sun L, et al. Early versus delayed hormonal therapy for prostate specific antigen only recurrence of prostate cancer after radical prostatectomy. J Urol 2004;171:1141-7.

12 Lowrance WT, Breau RH, Chou R, et al. Advanced prostate cancer: AUAVASTRO/SUO guideline Part I. J Urol 2021;205:14-21.

13 Crook JM, O'Callaghan CJ, Duncan G, et al. Intermittent androgen suppression for rising PSA level after radiotherapy. N Engl J Med 2012;367:895-903.

14 Loblaw DA, Virgo KS, Nam R, et al. Initial hormonal management of androgen-sensitive metastatic, recurrent, or progressive prostate cancer: 2006 update of an American Society of Clinical Oncology practice guideline. J Clin Oncol 2007;25:1596-605.

15 Tombal B, Borre M, Rathenborg P, et al. Enzalutamide monotherapy in hormone-naive prostate cancer: primary analysis of an open-label, single-arm, phase 2 study. Lancet Oncol 2014;15:592-600.

16 Tombal B, Borre M, Rathenborg P, et al. Long-term efficacy and safety of enzalutamide monotherapy in hormone-naïve prostate cancer: 1- and 2-year open-label follow-up results. Eur Urol 2015;68:787-94.

17 Tombal B, Borre M, Rathenborg P, et al. Long-term antitumor activity and safety of enzalutamide monotherapy in hormone naïve prostate cancer: 3-year open label follow-up results. J Urol 2018;199:459-64.

18 Armstrong AJ, Szmulewitz RZ, Petrylak DP, et al. ARCHES: a randomized, phase III study of androgen deprivation therapy with enzalutamide or placebo in men with metastatic hormone-sensitive prostate cancer. J Clin Oncol 2019;37:2974-86.

19 Chi KN, Agarwal N, Bjartell A, et al. Apalutamide for metastatic castration-sensitive prostate cancer. N Engl J Med 2019;381:13-24.

20 Fizazi K, Tran N, Fein L, et al. Abiraterone plus prednisone in metastatic, castration-sensitive prostate cancer. N Engl J Med 2017;377:352-60.

21 Davis ID, Martin AJ, Stockler MR, et al. Enzalutamide with standard first-line therapy in metastatic prostate cancer. $N$ Engl $\mathrm{J}$ Med 2019;381:121-31.

22 Hussain M, Fizazi K, Saad F, et al. Enzalutamide in men with nonmetastatic, castration-resistant prostate cancer. $N$ Engl J Med 2018;378:2465-74.

23 Sternberg CN, Fizazi K, Saad F, et al. Enzalutamide and survival in nonmetastatic, castration-resistant prostate cancer. $N$ Engl J Med 2020;382:2197-206

24 Armstrong AJ, Lin P, Tombal B. Five-year survival prediction and safety outcomes with enzalutamide in men with chemotherapy-naïve metastatic castration-resistant prostate cancer from the prevail trial 2020;78:347-57

25 Beer TM, Armstrong AJ, Rathkopf D, et al. Enzalutamide in men with chemotherapy-naïve metastatic castration-resistant prostate cancer: extended analysis of the phase 3 PREVAIL study. Eur Urol 2017;71:151-4.

26 Beer TM, Armstrong AJ, Rathkopf DE, et al. Enzalutamide in metastatic prostate cancer before chemotherapy. N Engl J Med 2014;371:424-33.

27 Scher HI, Fizazi K, Saad F, et al. Increased survival with enzalutamide in prostate cancer after chemotherapy. N Engl J Med 2012;367:1187-97. 
28 Taylor LG, Canfield SE, Du XL. Review of major adverse effects of androgen-deprivation therapy in men with prostate cancer. Cancer 2009;115:2388-99.

29 Keating NL, O'Malley AJ, Freedland SJ, et al. Diabetes and cardiovascular disease during androgen deprivation therapy: observational study of veterans with prostate cancer. J Natl Cancer Inst 2010;102:39-46.

30 Shahinian VB, Kuo Y-F, Freeman JL, et al. Risk of fracture after androgen deprivation for prostate cancer. N Engl J Med 2005;352:154-64.

31 Pisansky TM, Thompson IM, Valicenti RK, et al. Adjuvant and salvage radiotherapy after prostatectomy: ASTRO/AUA guideline amendment 2018-2019. J Urol 2019;202:533-8.

32 Abramowitz MC, Li T, Buyyounouski MK, et al. The Phoenix definition of biochemical failure predicts for overall survival in patients with prostate cancer. Cancer 2008;112:55-60.

33 Arlen PM, Bianco F, Dahut WL, et al. Prostate Specific Antigen Working Group guidelines on prostate specific antigen doubling time. J Urol 2008;179:2181-6. discussion 2185-6.

34 Keto CJ, Aronson WJ, Terris MK, et al. Detectable prostate-specific antigen nadir during androgen-deprivation therapy predicts adverse prostate cancer-specific outcomes: results from the search database. Eur Urol 2014;65:620-7.

35 Cornford P, van den Bergh RCN, Briers E, et al. EAU-EANM-ESTROESUR-SIOG guidelines on prostate cancer. Part II-2020 update: treatment of relapsing and metastatic prostate cancer. Eur Urol 2021;79:263-82.

36 Xie W, Regan MM, Buyse M, et al. Metastasis-free survival is a strong surrogate of overall survival in localized prostate cancer. $J$ Clin Oncol 2017;35:3097-104.

37 Twycross R, Harcourt J, Bergl S. A survey of pain in patients with advanced cancer. J Pain Symptom Manage 1996;12:273-82.

38 Cella DF, Tulsky DS, Gray G, et al. The Functional Assessment of Cancer Therapy scale: development and validation of the general measure. J Clin Oncol 1993;11:570-9.

39 Herdman M, Gudex C, Lloyd A, et al. Development and preliminary testing of the new five-level version of EQ-5D (EQ-5D-5L). Qual Life Res 2011;20:1727-36.

40 Smith MR, Saad F, Chowdhury S, et al. Apalutamide treatment and metastasis-free survival in prostate cancer. $N$ Engl $J$ Med 2018;378:1408-18. 